\title{
Anti-reflective sub-wavelength patterning of IR optics
}

D. Vandormael, S. Habraken, J. Loicq, C. Lenaerts, D. Mawet

D. Vandormael, S. Habraken, J. Loicq, C. Lenaerts, D. Mawet, "Antireflective sub-wavelength patterning of IR optics," Proc. SPIE 6395, ElectroOptical and Infrared Systems: Technology and Applications III, 63950L (5 October 2006); doi: 10.1117/12.692449

SPIE. Event: Optics/Photonics in Security and Defence, 2006, Stockholm, Sweden 


\title{
Antireflective subwavelength patterning of IR optics
}

\author{
D. Vandormael ${ }^{* a}$, S. Habraken ${ }^{a+b}$, J. Loicq $^{a}$, C. Lenaerts ${ }^{b}$, and D. Mawet ${ }^{b}$. \\ ${ }^{a}$ Centre Spatial de Liege, Av. Du Pre-Aily, B-4031 Angleur, Belgium ; \\ ${ }^{\mathrm{b}}$ University of Liege, Physics \& Astrophysics Departments, Bat. B5, B-4000 Liege, Belgium
}

\begin{abstract}
Thermal infrared (IR) lenses require efficient anti-reflection coating. Moth-eye (or egg-box) 2D subwavelength gratings have demonstrated their ability to reach a very high transmission for a wide wavelength and angular range. The use in thermal IR is simplified by the lower resolution for lithographic technology, compared to visible waveband. However, deeper structures must be engraved and lithography must be adapted to IR materials. In order to be cost-effective, the patterning must be produced by replication techniques, such as embossing. Our laboratory is now experimenting hot embossing of moth-eye patterns in chalcogenide substrates.

In this paper, theoretical analysis, micro-lithographic technology and manufacturing processes are detailed.
\end{abstract}

Keywords: antireflective, infrared, moth-eye, replication

\section{INTRODUCTION}

Fresnel reflections from surfaces in optical systems are cumbersome, particularly when there are many surfaces in the system: the total power loss can be considerable, and stray light due to the reflections tends to reduce the contrast in imaging systems. Optical coatings are the standard way to reduce reflections from optical surfaces, but it is expensive and unfortunately often a source of issues in numerous applications. Indeed, multilayer thin films show problems associated with limitations in the coating materials, especially in the infrared. Classical coatings also exhibit various physical and chemical side effects affecting adhesion, thermal mismatch and durability of the thin-film stack. These problems are of course exacerbated in thermal imaging applications, especially in space where stability constraints are emphasized. In 1967, Bernhard [1] discovered that the corneas of night-flying moths was covered with a fine regular hexagonal array of protuberances, which have a period of about $200 \mathrm{~nm}$ and a similar depth with cross section that is approximately sinusoidal. This geometry induces a natural index gradient which was identified as a natural mean to reduce the reflection over a wide spectral and angular bandwidth, improving the moth's camouflage. This principle is the same as the one used for anechoic chambers. The purpose of this paper is the implementation of moth-eye structures on the surface of infrared chalcogenide components as an alternative to the classical antireflective (AR) coatings. The design, the sample manufacturing and the analysis of experimental results will be presented.

\section{THEORETICAL DESIGN}

Unwanted Fresnel reflection comes from the sudden transition from an optical medium of refractive index $n_{a}$ to another one characterized by a different index $\mathrm{n}_{\mathrm{b}}$. The fraction $\mathrm{R}$ of light intensity reflected is given at normal incidence by the well-known Fresnel relation:

$$
R=\frac{\left(n_{b}-n_{a}\right)^{2}}{\left(n_{b}+n_{a}\right)^{2}}
$$

If, like on the moth's eye, there is a gradual change of index at the interface between two different materials, the net reflectance can be seen as the resultant of an infinite series of infinitesimal reflections at each incremental change of

*dvandormael@ulg.ac.be; phone+32436766 68; fax +32336756 13; www.csl.ulg.ac.be

Electro-Optical and Infrared Systems: Technology and Applications III, edited by Ronald G. Driggers,

David A. Huckridge, Proc. of SPIE Vol. 6395, 63950L, (2006) · 0277-786X/06/\$15 · doi: 10.1117/12.692449 
index. Since the reflection comes from a different depth from the surface, each will have a different phase. If the transition takes place over at least a significant fraction of a wavelength, the resultant total reflection can be significantly reduced because all phases will be present and destructively interfere with each other [2]. This sort of "impedance matching" between the incident and substrate indices can be implemented via two methods: the quarter-wave transformer design and the graded or tapered index design [3].

The quarter-wave transformer design requires a stack of discrete index layers on a substrate, where the depth of each layer is a quarter wave in the layer and the index of refraction of each layer is designed with use of the optimal Tschebyscheff synthesis technique. The resultant interference coating produces a bandpass filter, where the bandwidth of the passband region and the maximum threshold level depend on the number of discrete quarter-wave layers placed on the substrate.

The graded index matching design requires to continuously varying the refractive index from the incident region into the substrate region. The length of the taper and the index distribution function are determined with use of the optimal Klopfenstein tapering technique [4]. This particular graded index surface will produce a high-pass or short-wave filter. The cut-off frequency is the lowest frequency (longest wavelength) at which the reflectivity is below the desired maximum threshold level.

In the frame of this work, we have chosen the rigorous coupled-wave analysis (RCWA) as a powerful numerical tool to simulate typical performances of moth-eye structures [5]. Our guideline for the performance optimization of subwavelength grating anti-reflective structures is extremely pragmatic and in direct relation with the most appropriate manufacturing processes. We will indeed consider and simulate two types of gratings. According to traditional photolithographic processes, which naturally produce binary rectangular structures, we will consider rectangular profiles on one hand. According to holographic recording technique, which naturally produces sinusoidal profiles, we will consider sinusoidal profiles on the other hand.

\subsection{Rectangular profile design}

Figure 1 shows simulations performed for a typical infrared material, Umicore GASIR-1 ${ }^{\mathrm{TM}}$, patterned with a 2D rectangular profile subwavelength grating. As shown on the figure, the calculated average transmittance is $99 \%$ in the long-wave infrared (LWIR) and $98.5 \%$ in the mid-wave infrared (MWIR) bands, instead of $82 \%$ for an untreated single interface $(\mathrm{n} \sim 2.49)$.
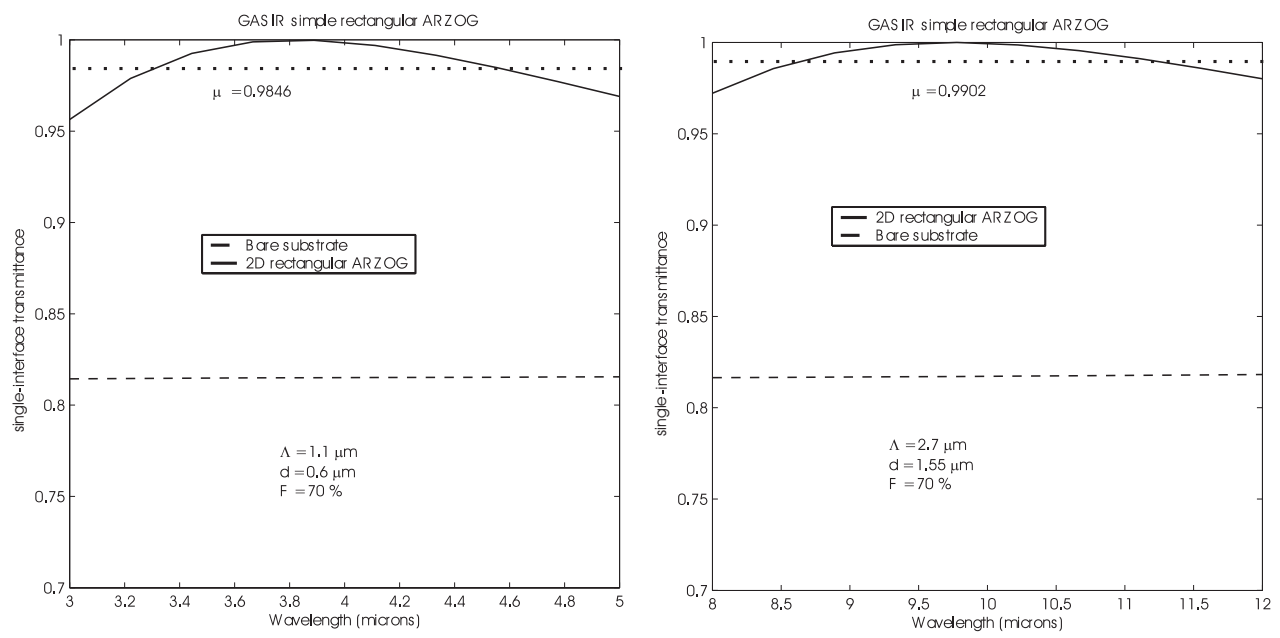

Figure 1. Simulation of the AR efficiency for one treated interface vs. wavelength in the thermal IR (Legend: ARZOG : antireflective zeroth order grating, F : Filling Factor, $d$ : thickness, $\Lambda$ : period, material : Gasir ${ }^{\mathrm{TM}}$ chalcogenide).

The simple rectangular profile in fact corresponds to a single quarter-wave layer with an effective index depending on the filling factor. By appropriately tuning the filling factor, the layer effective index can be chosen to provide the exact impedance matching between the substrate and the incident medium. This can lead to a substantially better performance 
than with a traditional single-layer treatment where the choice in actual material is always limited. The tolerance of simple rectangular treatments with respect to parameter changes is critical and, for thermal infrared applications, is at the 100-nm level for thickness and feature line control. Moreover, sensitivity to incidence variation for simple rectangular profiles is of a few degrees only. It is not comparable to the moth-eye profile performance, which, by realizing a perfectly smooth transition, decreases the parasitic reflection down to the percent level over huge bandwidths, as shown in next section.

\subsection{Sinusoidal profile design}

Simulations were carried out on the basis of a profile resulting from the intersection of two sinusoidal crossed gratings. Figure 2 shows the calculated antireflective efficiency for two variants of a so-called egg-box structure in Umicore GASIR-1 ${ }^{\mathrm{TM}}$ material. Figure 4 (left) shows the corresponding simulation for IG-6 ${ }^{\mathrm{TM}}$ chalcogenide (n 2.78).
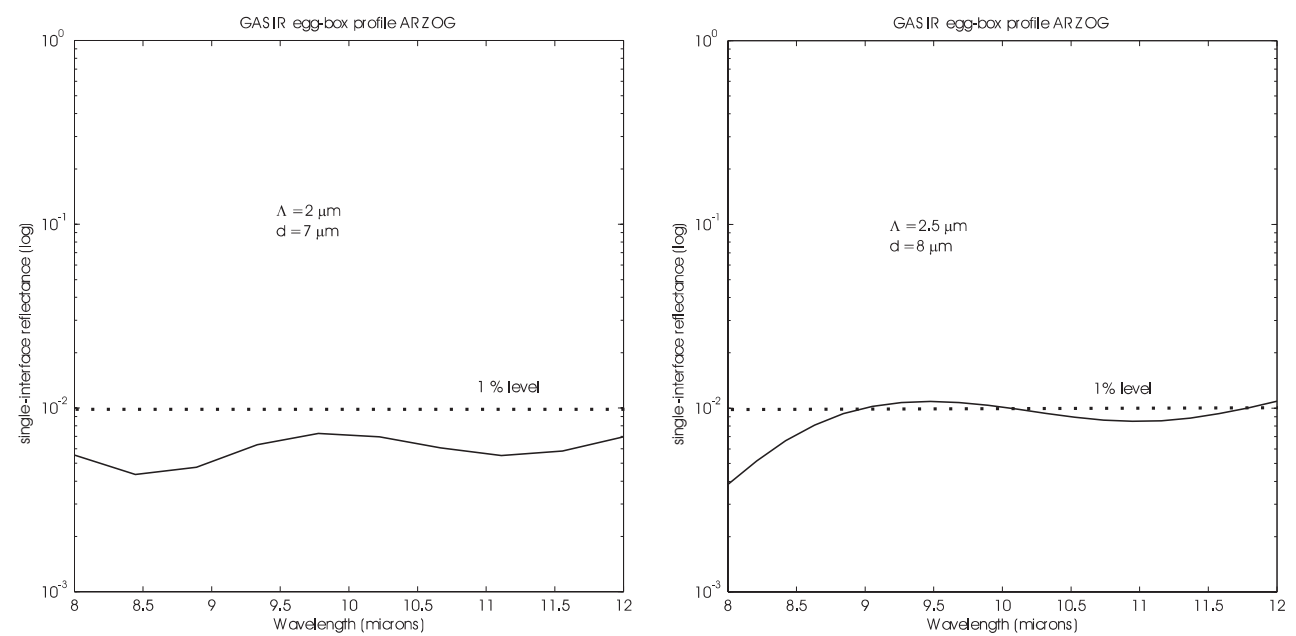

Figure 2. Simulation of the AR efficiency for one treated interface vs. wavelength in the thermal IR for 2 variants of egg-box structures (Legend: ARZOG : anti-reflective zero order grating, F : Filling Factor, $d$ : thickness, $\Lambda$ : period, material : Gasir ${ }^{\mathrm{TM}}$ chalcogenide).

Figure 3 shows the dependence of the calculated efficiency with respect to the profile depth for a $3 \mu \mathrm{m}$-period egg-box profile in Gasir-1 ${ }^{\mathrm{TM}}$, in the LWIR region.

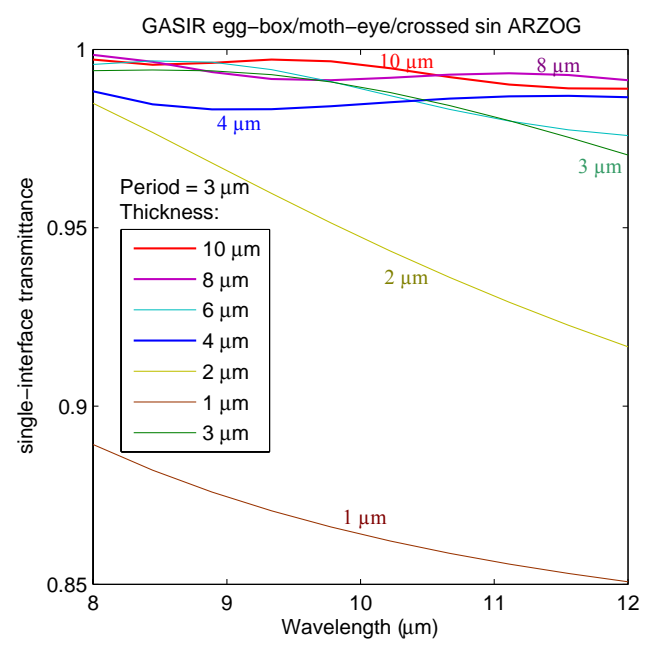

Figure 3. Single-interface AR efficiency vs wavelength in the thermal IR (simulation) for a 2D egg-box structure for various profile thicknesses. 
One can see that deeper structures provide the higher efficiency. Actually, the response of the AR structure for thermal infrared applications to a change in the total depth of the grating in the case of a moth-eye profile is sensitive at the level of several microns. Concerning the tolerance to incidence angle variations, the well-known wide acceptance of such structure is verified (Figure 4, right). However, it is clear that the structure should be optimized for the chosen working incidence.
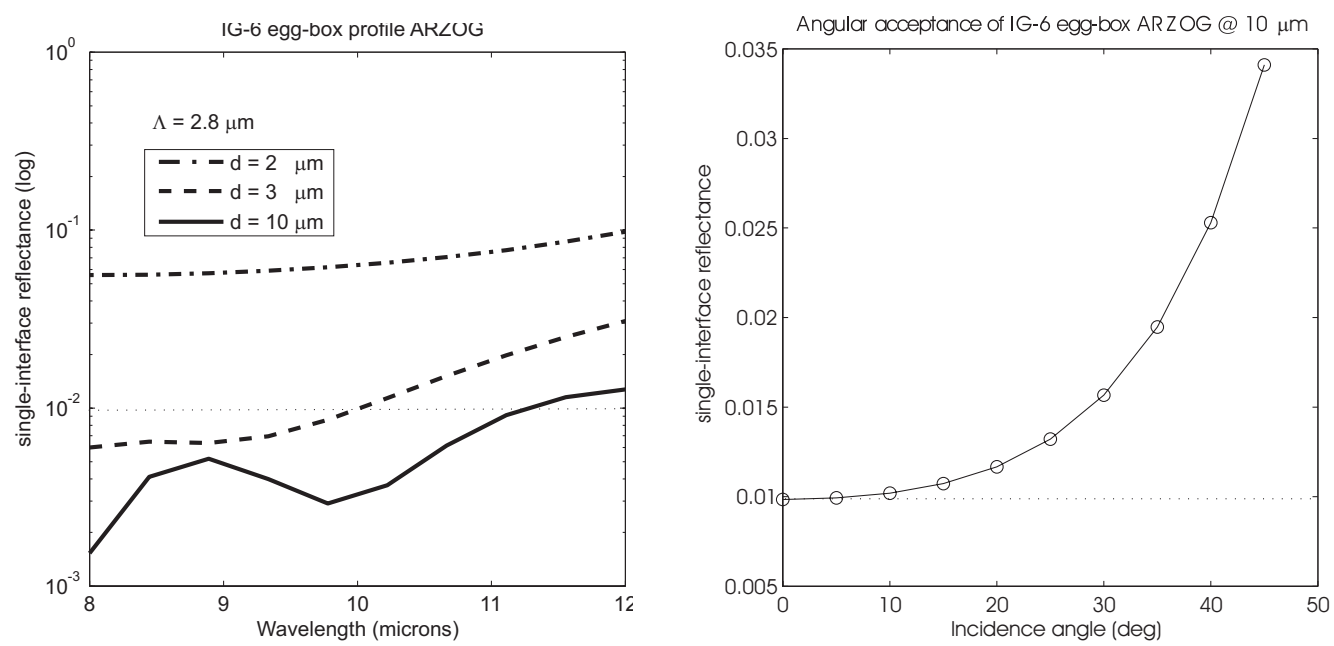

Figure 4. Left: residual reflectivity for a single interface vs. wavelength in the thermal IR for 3 different thicknesses of the egg-box structures. Right: angular acceptance of the egg-box profile ARZOG in the IG-6 case at 10 microns. Legend: ARZOG : antireflective zero order grating, $\mathrm{F}$ : Filling Factor, $\mathrm{d}$ : thickness, $\Lambda$ : period, material : IG- $6^{\mathrm{TM}}$ chalcogenide.

\section{EXPERIMENTAL}

Different fabrication methods have been identified. They are based on classical microfabrication techniques on one hand and the availability of new chalcogenide-based infrared glasses on the other hand. Those materials are very promising because of their excellent optical properties in the thermal IR band but also due to their molding ability, thanks to their glass transition temperature below $300^{\circ} \mathrm{C}$. Bulk molding process has already been implemented for cost-effectively manufacturing commercially available products. On the other hand, surface embossing appears to be another promising replication technique for patterning IR optical elements according to a well-defined surface pattern, such as an antireflective moth-eye structure. Our laboratory is now experimenting hot embossing of moth-eye patterns onto chalcogenide materials.

The manufacturing process consists in three main steps: pattern origination by photolithography, master fabrication by electrolysis, and final pattern transfer by hot embossing.

Rectangular profile micro-structure is originated by Direct Laser Writing (DLW). A laser beam is used as a controlled writing tool for generating patterns on a photosensitive material (photoresist). Writing pattern is performed by accurately moving the target (substrate) underneath the focused and scanning laser beam with appropriate wavelength. Sine profile micro-structure is obtained by holography, which consists in recording the contrast pattern of two interfering laser beams. An appropriate structure is obtained by recording two 1D sinusoidal gratings, flipped by 90 degrees one to the other. In both cases, this exposure step is followed by a chemical processing in order to remove the resist from the exposed areas. The previously contrasted illuminating pattern is therefore transformed into a relief structure. Figure 5 shows a scanning electron microscope (SEM) photography of the resulting photoresist pattern obtained by DLW and by holography. 

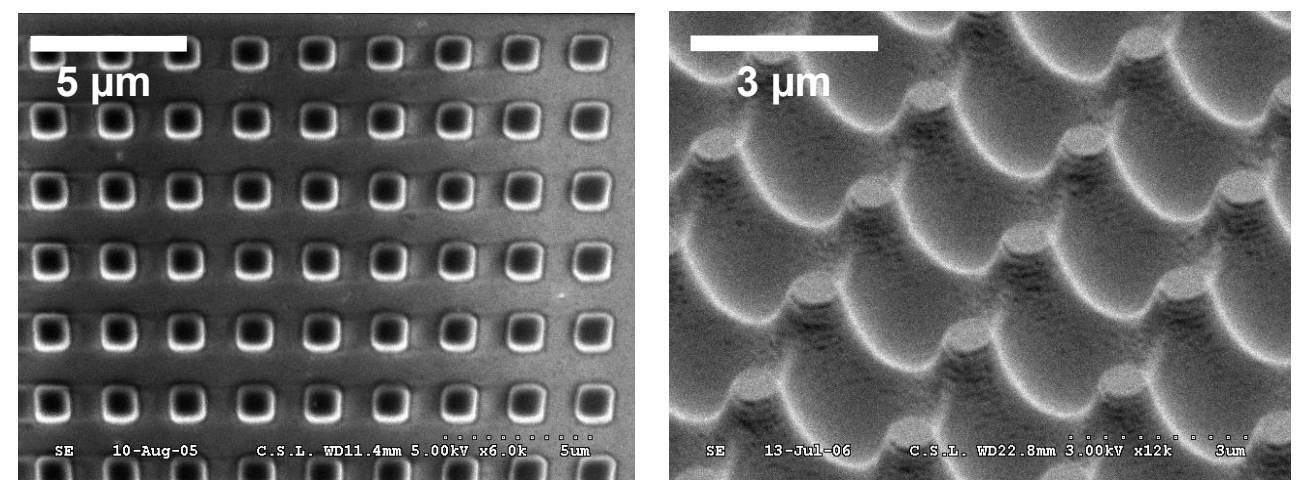

Figure 5. Photoresist pattern originated by DLW (left, view from the top) and holography (right, oblique view).

An electroplating process is then needed to grow a metallic master (shim) from the photoresist structure. The resulting imprint is hard enough to be used in a hot embossing process where it will be heated and pressed on the surface of the mouldable material in order to transfer the microstructure. Figure 6 shows a detail of the negative metallic master obtained from the rectangular and the sine profile structures, respectively.
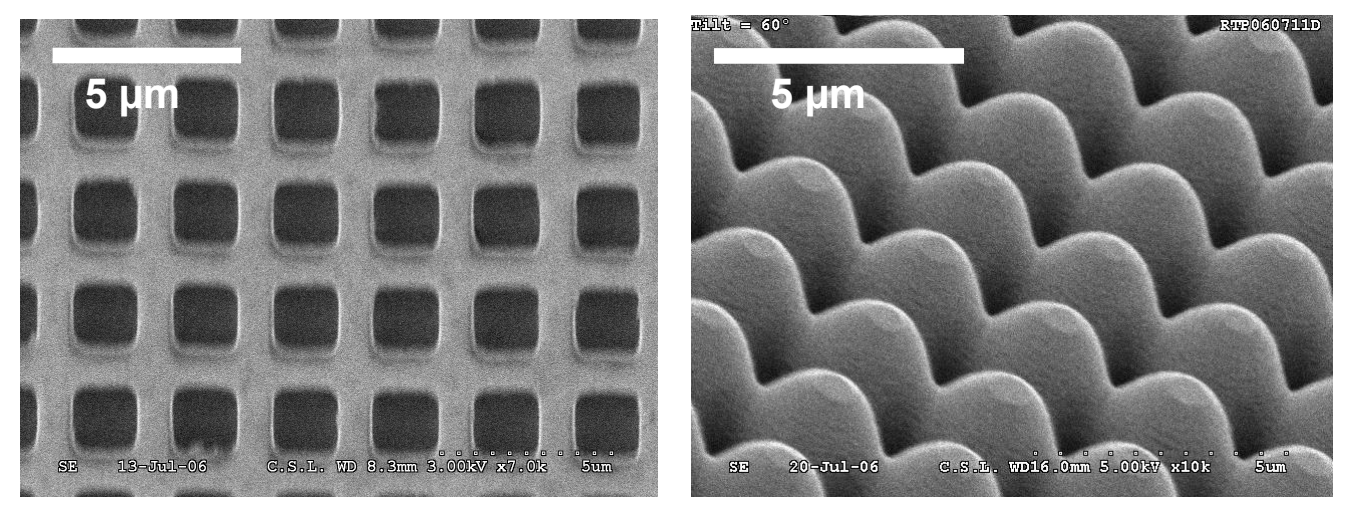

Figure 6. SEM picture of a metallic master obtained by electrolysis from a rectangular (left) and sinusoidal (right, oblique view) profile pattern

Next step consists in transferring the pattern from the metallic master into the chalcogenide material, thanks to an appropriate thermal and pressure cycle. This operation is performed with a double-jack, flat-bed, hydro-pneumatic heating press. The equipment has been previously used for replicating diffractive structures into moldable polymer materials. This manufacturing technique is now under experiment on the basis of commercially available chalcogenide blanks (Gasir ${ }^{\mathrm{TM}}$ by Umicore and $\mathrm{IG}^{\mathrm{TM}}$ by Vitron). Figure 7 shows the resulting pattern obtained by embossing the microstructured profile onto the surface of a Vitron IG- $6^{\mathrm{TM}}$ window.
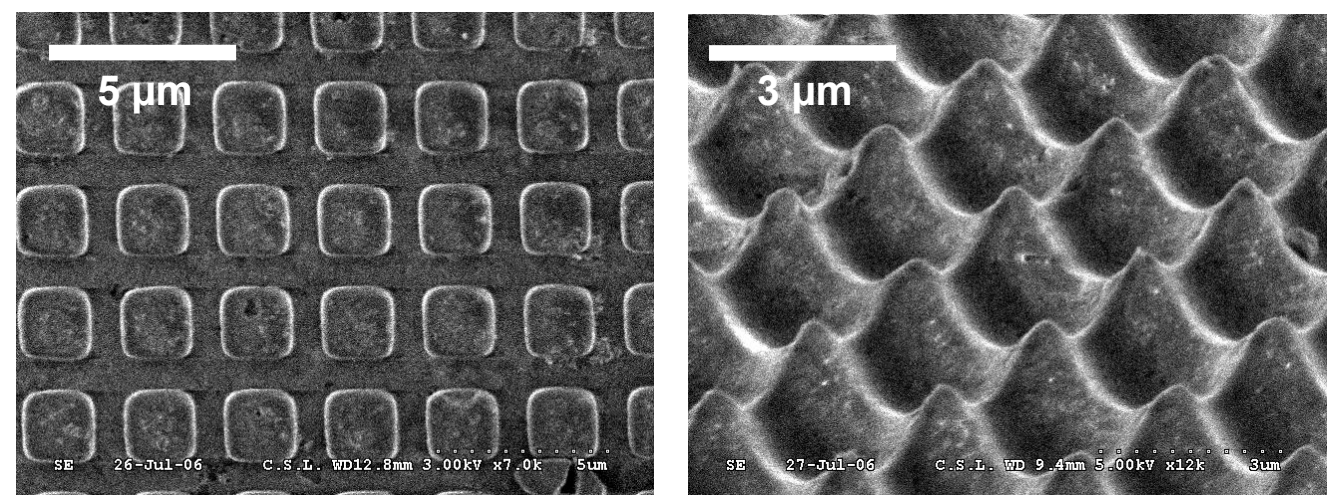

Figure 7. Rectangular (left) and sinusoidal (right) profile structures embossed onto Vitron IG-6 ${ }^{\mathrm{TM}}$ surface. 
Thanks to the more relaxed tolerances of the design, the sine profile structure is much less sensitive to shape modifications which might occur from the process. Moreover, in any kind of replication methods, smooth details are reputed to be transferred with a lower degree of damage compared to sharp and vertical features. Indeed these latter are more subjected to sticking effects and alteration during mould separation. Nevertheless, for both structures, surface roughness and microstructure degradation still needs to be minimized.

\section{RESULTS AND DISCUSSION}

Transmission measurements have been performed on the basis of a sine profile moth-eye structure embossed onto Vitron IG-6 ${ }^{\mathrm{TM}}$ plano-disc samples. As depicted in Figure 7, the replicated moth-eye structure exhibits a period of $2.8 \mu \mathrm{m}$ and a peak-to-valley depth around $2.0 \mu \mathrm{m}$ (SEM observations). Two types of measurements were performed with:

- a FTIR (Fourier-Transform IR) spectrometer, and

- $\quad \mathrm{a} \mathrm{CO}_{2}$ laser emitting at $10.6 \mu \mathrm{m}$ wavelength.

Figure 8 shows the behavior of the samples based on FTIR measurements. Three samples are compared:

- a plano disc (polished blank) of IG-6 material,

- a flat embossed disc, with no microstructure on its faces,

- a flat embossed disc, with a moth-eye structure replicated on one of its faces (1-side moth-eye).

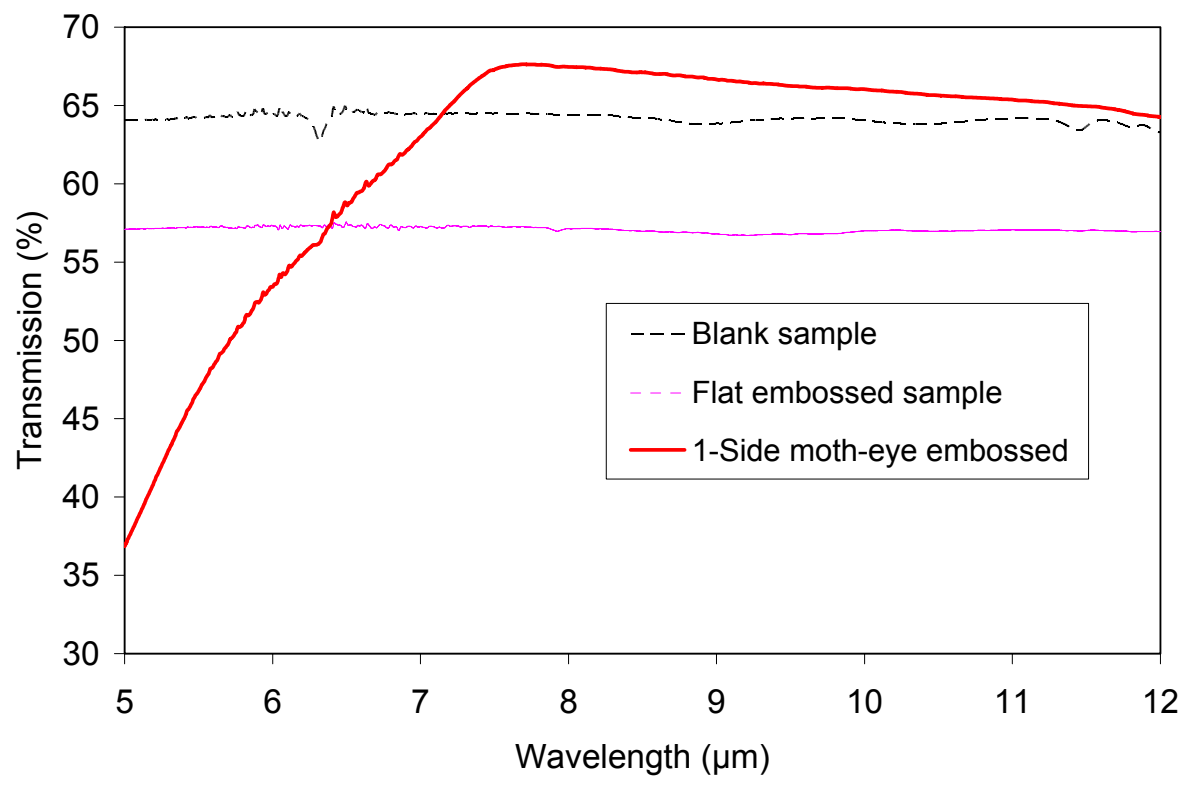

Figure 8. FTIR measurement of transmission through IG-6 samples.

The low wavelength range behavior clearly shows that the periodic structure was optimized for wavelength higher than $8 \mu \mathrm{m}$. The transmission loss of the moth-eye plate results from emerging diffraction orders. Above $8 \mu \mathrm{m}$, the zero ${ }^{\text {th }}$ orders only are allowed to propagate (reflection + transmission). The usable range shows that, for unknown reasons, the blank disc exhibits a transmission higher than theoretically expected. With an average refractive index of 2.78, the Fresnel reflection should be $\mathrm{r}_{\text {air-IG6 }}=22.15 \%$ per air-IG6 interface. With two interfaces, the disc should therefore exhibit a reflection of $R=R_{1}+R_{2}=r(2-r)=39.4 \%$ or equivalently a resulting transmission of $60.6 \%$, lower than the $64 \%$ we measured instead.

The flat embossed sample exhibits a measured transmission of 57\%, which means a loss of about $7 \%$ with respect to the blank value. This is either attributed to the known refractive index increase of chalcogenide molded samples, and/or to scattering due to surface roughness degradation during the embossing process. 
The single side moth-eye embossed sample shows a transmission much higher (66-67\%) than the flat embossed one. We can expect that the index increase or the scattering issue is identical in both cases, as we can imagine from the SEM observations (Figure 7). However, the transmission is higher than the blank original, but only a few percents.

The transmission measurement based on $\mathrm{CO}_{2}$ laser line at $10.6 \mu \mathrm{m}$ was needed to validate the results. Measurements at various small angles were compiled in order to get rid of interferential fluctuations coming from spurious interface reflections. The plano-disc (blank) sample verifies the expected theoretical value (61\%). The flat embossed sample exhibits a transmission of $57 \%$, which confirms the FTIR measurement. The moth-eye embossed sample exhibits a transmission of $70.5 \%$, which is much higher and encouraging.

Based on simulation of Figure 4, with $2 \mu \mathrm{m}$ thickness, the moth-eye residual reflectance is $7 \%$. Combined with the flat interface reflectance $(22.15 \%$ ), we find a theoretical transmission through the moth-eye (single side) sample of $72.4 \%$. This has to be compared with the measurement $(70.5 \%)$. Once again, the loss is certainly due to the index increase and/or scattering. We measure a lower scattering effect compared to the FTIR value, which is probably related to the proximity of the detector to the sample (part of the scattered light is still collected by the detector).

We feel strongly confident that improvement of the embossed process will allow to dramatically reduce the scattering. Anti-sticking solutions will be investigated in order to optimize the separation between the master and the material. Moreover, our next efforts will also be directed to a deeper structure using deeper masters. Figure 4 shows that a thickness of $3 \mu \mathrm{m}$ would significantly improve the efficiency. This goal could be achieved thanks to an intermediate fabrication step where a photolithographically recorded profile is transferred by dry etching into another material. According to an appropriate choice of the material selectivity (the etching rate of the bulk material with respect to the photoresist mask), profile shapes can be conserved while increasing their depth. Alternative photolithographic techniques, such as excimer laser ablation, might also help in etching deeper structures into materials compatible with the subsequent master fabrication process. Embossing both sides of the sample with moth-eye structures is also our nearterm goal, in order to prove the efficiency of the concept and process.

\section{CONCLUSIONS}

This paper has presented preliminary results of anti-reflection structuring onto chalcogenide IR compounds. The thermoforming characteristics of the materials were used to pattern the surface with moth-eye structures. A manufacturing method based on the surface embossing of the bulk material with a hot master originated by photolithography and electrolysis has been investigated. We have demonstrated the theoretical concept and validated it through experimental results. In the near future, improved process should help us to demonstrate that this kind of treatment is able to compete with traditional AR coatings, as a cost-effective technique.

\section{ACKNOWLEDGEMENTS}

CSL acknowledges the Walloon region for financial support through the Euclid/Europa program.

\section{REFERENCES}

1. Bernhard, G., "Structural and functional adaptation in a visual system", Endeavour 26, p. 79, 1967.

2. Wilson, S. \& Hutley, M., "The optical properties of "moth eye" antireflection surfaces", Optica Acta 29, pp. 993-1009, 1982.

3. Collins, R. E., "Foundations for Microwave Engineering", chap. 5 (Mc-Graw Hill, Inc.), 1966.

4. Klopfenstein, R. W., "A transmission line taper of improved design”, in Proc. IRE 44, 1956.

5. Moharam, M. \& Gaylord, T., "Three dimensional vector coupled-wave analysis of planar grating diffraction", J. Opt. Soc. Am. 73, pp. 1105-1112, 1983. 